\title{
REAL TIME BIOSIGNAL-DRIVEN ILLUSION SYSTEM FOR UPPER LIMB REHABILITATION
}

\author{
Yee Mon Aung, Adel Al-Jumaily \\ Faculty of Engineering and Information Technology, University of Technology Sydney \\ 15 Broadway Ultimo NSW 2007, Australia \\ yee.m.aung@student.uts.edu.au, adel.al-jumaily@uts.edu.au
}

\begin{abstract}
This paper presents design and development of real time biosignal-driven illusion system: Augmented Reality based Illusion System (ARIS) for upper limb motor rehabilitation. ARIS is a hospital / home based selfmotivated whole arm rehabilitation system that aims to improve and restore the lost upper limb functions due to Cerebrovascular Accident (CVA) or stroke. Taking the advantage of human brain plasticity nature, the system incorporates with number of technologies to provide fast recovery by re-establishing the neural pathways and synapses that able to control the mobility. These technologies include Augmented Reality (AR) where illusion environment is developed, computer vision technology to track multiple colors in real time, EMG acquisition system to detect the user intention in real time and 3D modelling library to develop Virtual Arm (VA) model where human biomechanics are applied to mimic the movement of real arm. The system operates according to the user intention via surface electromyography (sEMG) threshold level. In the case of real arm cannot reach to the desired position, VA will take over the job of real arm to complete the exercise. The effectiveness of the developed ARIS has evaluated via questionnaire, graphical and analytical measurements which provided with positive results.
\end{abstract}

\section{KEY WORDS}

Upper limb rehabilitation, illusion system, augmented reality, surface electromyography.

\section{Introduction}

Stroke or Cerebrovascular Accident (CVA) is the second most common fatal disease and leading cause of disability in Australia. According to National Stroke Foundation, over 420,000 Australians were living with the effects of stroke in 2012. This was over $1.77 \%$ of the Australian population and it will increase to $2.4 \%$ of the Australian population by 2032 [1]. Stroke takes place due to the formation of plaque in the blood vessels. Plaque is built up of fat, cholesterol, calcium and other substances from blood. It will precipitate to the lumen of blood vessels and become thicker and harden over a period of time, and then it starts to restrict the blood flow. When blood flow is interrupted by blood clot or ruptured blood vessels, the supplement of oxygen to the brain will be disturbed causing brain cell death and this will lead to stroke. Due to dead brain cells, some of the brain functions cannot work properly such as impairment in cognitive, communication, sensory perceptual and physical activities depending upon which part of the brain is damaged. To recover such impairments, rehabilitation programme are conducted in hospitals and clinics by a rehabilitation team that includes staff with different skills. Rehabilitation is the process of training to recover or improve lost functions due to injury or illness. It should start as soon as stroke patients are stabilised with medical condition and should end only when there is no sign or symptom of stroke. Rehabilitation will provide patients to reestablish the neural pathways and synapses that able to control the mobility by oneself due to the nature of human neuroplasticity. According to physiotherapy board of Australia, only 23,301 people were registered as physiotherapists by 2012 and this is a ratio of $12: 1$ (Patients vs. Therapist) [1]. This leads to shortage of physiotherapist and become one of the major problems in Australia currently. Another major problem is the economic impact in Australia due to stroke. In 2012, about AU $\$ 50$ billion were used in burden of disease costs for stroke.

To overcome above mentioned major problems due to stroke, researchers are trying to develop low cost rehabilitation system which requires less supervision by therapist. Such developments include robotics assistive rehabilitation systems and non robotics assistive systems. However, robotics assistive systems [2, 3] consider as an expensive approach due to its hardware development cost although the better recovery result were provided for major impairment. Therefore, developments of the non robotics assistive system such as Virtual Reality (VR) based rehabilitation systems [4] and Augmented Reality (AR) based rehabilitation systems $[5,6]$ become popular because they provide better motivation by integrating with games like exercises as rehabilitation exercises. In addition to that, researchers have integrated with biofeedback system to VR [7] or AR system [8] or even with robotics assistive systems [2,9] to provide fast recovery and studies have proven with positive results via clinical trials [10]. Biofeedback is a process that enables an individual to learn how to change the physiological for 
the purposes of improving health and performance. It allows to monitor the various physiological activities such as brainwaves via Electroencephalography (EEG), muscle tone through Electromyography (EMG), skin conductance via Electrodermograph (EDG) and heart rate with Electrocardiograph (ECG).

Another type of low cost approach for upper limb rehabilitation is called Mirror Therapy (MT). In MT treatment, patient is asked to sit in front of the mirror that is put between left and right arm. The mirror side is faced towards unaffected arm while impaired arm is hid behind the mirror. Therefore, when patient looks into the mirror, he/she will see the reflection of the unaffected arm as his/her impaired arm by creating the visual illusion. This approach has been used to treat the patients suffering from phantom limb pain (PLP) $[11,12]$, complex regional pain syndrome (CRPS) [12, 13] and stroke [12, 14]. Integrating visual illusion provides fast recovery in rehabilitation due to human neuroplasticity ability which is mediated by beliefs, perceptions and emotions that react in everchanging ways, through thoughts and activities based on environmental input. The proven results of MT have reported in [15].

However, up to our knowledge, there are no developments have done for user intended illusion with AR based rehabilitation exercise to provide fast recovery hospital / home based therapy system. Therefore, the very first and low cost upper limb rehabilitation system called Augmented Reality based Illusion System (ARIS) has designed and developed with user own intention. The system integrates with computer vision technology, AR technology and real-time biofeedback with ownership illusion technique for fast recovery by making used of human neuroplasticity.

This paper is organized as follows: section 2 describes the ARIS framework and its implementations. Section 3 details the materials and methods of ARIS.
Section 4 depicted the results discussion of the experiments and finally, in section 5, conclusion is presented.

\section{ARIS Framework}

The developed ARIS aims to rehabilitate the upper limb motor deficiency by creating ownership illusion for fast recovery. The complete framework of ARIS such as architecture and technologies that employ in ARIS is as portrayed in Figure 1. The main framework is developed in Flash Professional CS6 platform with the help of exported data from Matlab. First, the sEMG data from user / patient are recorded via sEMG acquisition device and send to Matlab to process it. The processed data will then send to Flash Professional for further process in ARIS such as detection of user intention. The function of USB webcam in ARIS is to capture the live video to create AR environment and at the same time tracking the multiple color markers to create the illusion scene. In addition to this, graphical measurement such as real-time trajectory graph and analytical measurements such as sEMG activation level and kinematics data can be monitored and evaluated the user's progress during the rehabilitation process in ARIS.

\subsection{Architecture}

The system begins with the choice of left or right arm exercise depending on which side of the arm is paralyzed. Step by step information will be provided to the user of the display screen for ease of understanding on how to operate the system. After the rehabilitation exercise has selected, the appropriate exercise will display to the user with live video image, two buttons: capture button and

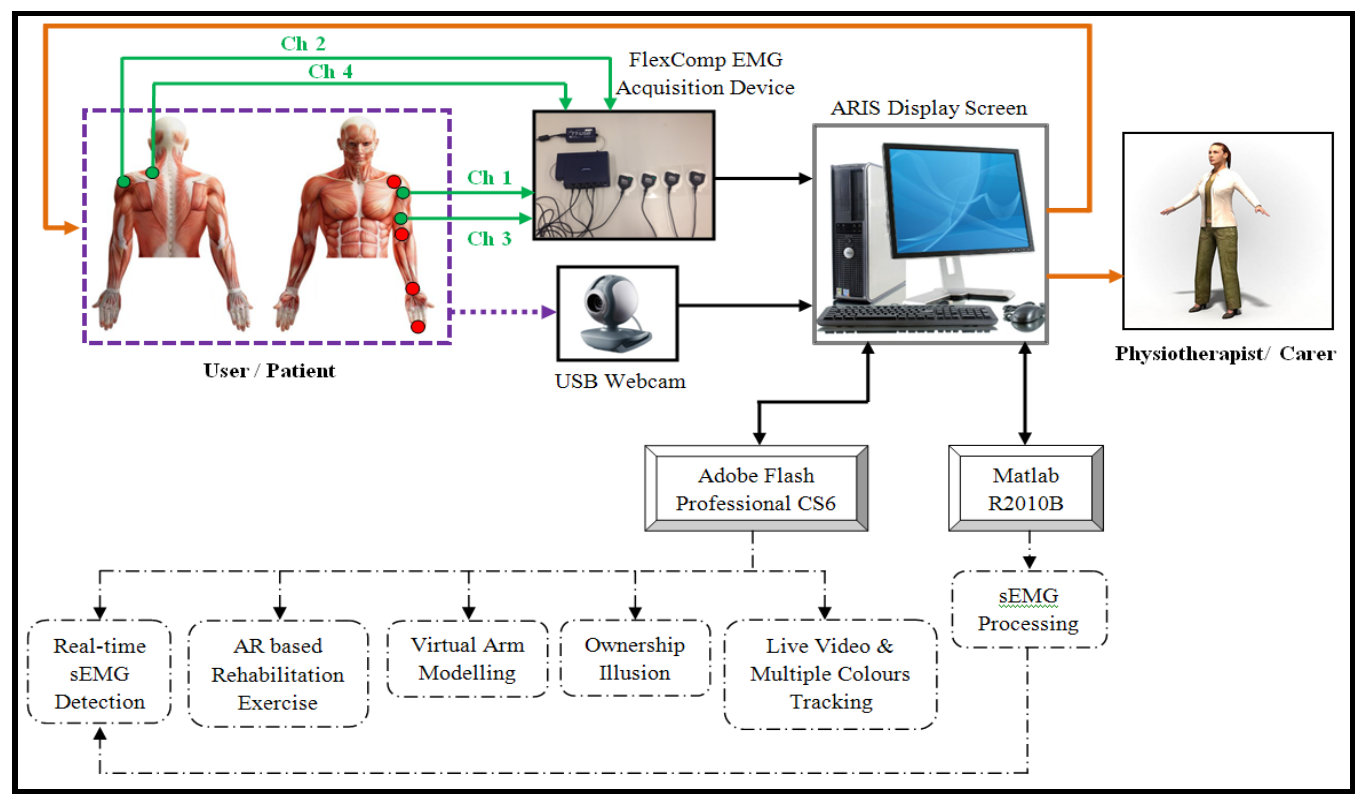

Figure 1. ARIS framework and its implementations (Red dot: Color Marker Position \& Green dot: Electrode Sites) 
start button, user key-in box for sEMG threshold level, real-time trajectory graph and real-time kinematics data. Before starting the exercise, the user is required to capture the current background image via capture button. This image will then store in the system and will use in the later stage to integrate as illusion scene. After that the system will request the user to choose the skin color by just clicking on the user's own skin color seen via webcam. At the same time, the VA model will be loaded within the system with selected skin color but would not be displayed to the user. Once the skin color has chosen the color markers which attach at the shoulder joint, elbow joint, wrist joint and finger tip will be asked by the system to track the position in real time. After all the colors have chosen, the VA model will be displayed as ownership illusion to the user and ready for the simulation. The activation of the model simulation depends on the sEMG threshold level that measured before the exercise with FlexComp EMG acquisition device [16]. This measured threshold value will be typed in by user, therapist or carer in ARIS system. The threshold level is user dependent and this level will determine the user intentions: above this threshold level, user has intention to move and therefore VA model will perform simulation or else the VA model will remain unchanged. In addition to this, this threshold value is one of the measurements to evaluate the muscle performance over rehabilitation period. After providing the required information to the system, user starts the rehabilitation exercise by pressing start button. Both real arm and VA model will start to move. The movement of the real arm will be completed by user own effort and this effort can be observed in real-time under real-time trajectory graph as well in $\mathrm{X}$ and $\mathrm{Y}$ position under real hand position. Therefore real time trajectory graph and sEMG threshold level are two types of measurements to evaluate the user reaching performance. In the case of real arm cannot reach to the desired position during the rehabilitation exercise, VA will take over the job of real arm by picking up the virtual object and placing at respective position. VA model moves according to the predefined trajectory by means of forward and kinematics formulations. The angle information of each VA joint is able to monitor in real-time.

\subsection{Implementation}

The main framework is developed in Flash Professional CS6 platform. Capturing of live video scene to generate AR environment, search for suspected color markers, and detect the collision between color marker and virtual objects are implemented with ActionScript 3 in Flash. Loading of 3D VA model with its associated kinematics information is completed through Papervision 3D API. Real time sEMG data is collected via Matlab platform and exported into Flash platform in real time. The complete system of ARIS is made up of five modules: live video and multiple colors tracking module, ownership illusion module, Virtual Arm (VA) model module, Augmented
Reality (AR) based rehabilitation exercise module and real-time sEMG module as illustrated in Figure 1.

\subsubsection{Live video and multiple colors tracking module}

This module is developed to realize the AR environment with multiple color tracking. The AR environment is generated by means of low cost webcam from personal desktop computer or built in webcam from laptop. The webcam captures the live image background in real time and display as an AR environment to the user. There are four color markers attached to the real arm which located at shoulder joint, elbow joint, wrist joint and tip of the finger as shown in Figure 2. Each of those color markers will be tracked via webcam to detect the current position of the user joint in real time. The tracking of the color marker is realized by color pixel value which is selected by mouse click.

\subsubsection{Ownership illusion module}

Ownership illusion means illusion of owning either part of the body or whole body which is normally overridden

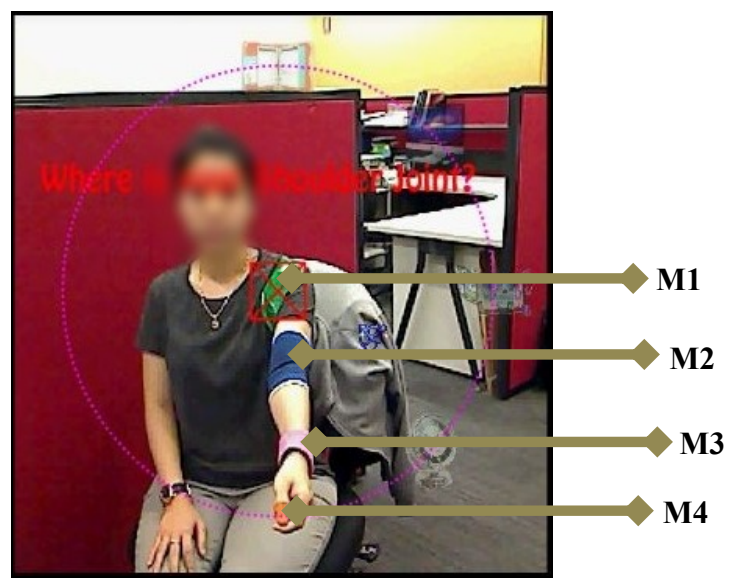

Figure 2. Location of color markers (M1: Green, M2: Blue, M3: Pink, M4: Orange)

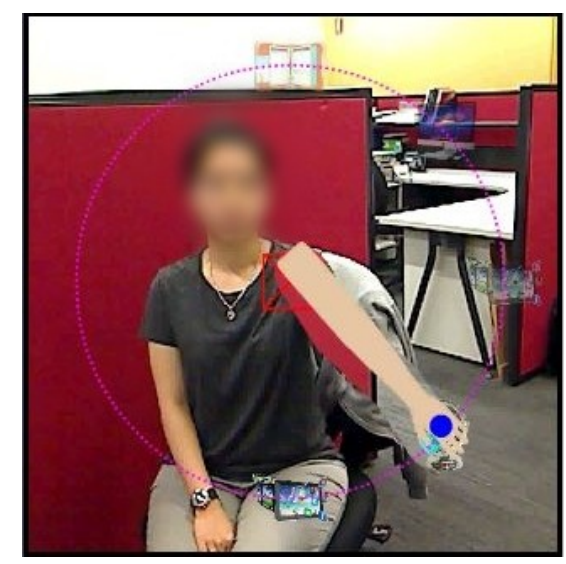

Figure 3. Ownership illusion concept (real arm is hidden and virtual arm is overlaid) 
by a virtual model. In this work, Virtual Arm (VA) model is created for ownership illusion scene to visualize the disappearance of real arm and then overlap with real arm like VA model as shown in Figure 3. The disappearance of the real arm is completed by covering with oval shape object which is filled with current background image that taken by webcam. The oval shape object is created with three segments that follow the human arm segments which are the upper arm, forearm and hand. The first segment is defined from shoulder to elbow color marker which is attached to shoulder joint and elbow joint. Similarly, second segment is defined from elbow color marker to wrist color marker and third segment is defined from wrist color marker to finger tip color. Therefore, on the display screen, wherever the real arm is moving at any joint angle, it will be fully covered with current background as a disappearance of real arm. Afterwards, the shoulder of real arm like VA model will be attached at the shoulder color marker to create the illusion scene as if user is seeing his/her own arm. The detail development of the VA model is described in section 2.2.3.

\subsubsection{Virtual Arm (VA) model module}

The arm like VA model is developed in Solidworks and exported as .dae model. The exported model is then imported to the flash via Papervision 3D class library which is an open source real time $3 \mathrm{D}$ engine to create the illusion scene as shown in Figure 3. There are 6 DoFs in VA model where 3 DOFs at shoulder joint, 1 DOF each at elbow, forearm and wrist joint respectively. The model segments and joints are properly defined to make sure the realistic movements during simulation. The mathematical modelling of the VA is formulated based on DenavitHartenberg (D-H) convention and detail of this development can be found in [17]. The allowable range of motion of the VA model is limited to safe articulation of the human arm according to [18]. The simulation of VA model is realised by forward and inverse kinematics calculation according to the predefined trajectory in rehabilitation exercise.

\subsubsection{AR based rehabilitation exercise module}

In ARIS, reaching exercise by picking and placing of virtual objects is chosen as an upper limb rehabilitation exercise because reaching exercise is the most common and effective rehabilitation exercise in rehabilitation hospitals and centres [18]. The ARIS exercise is versatile as it allows the user to choose either left or right arm training as shown in Figure 4. Therefore, the developed system is able to rehabilitate both sides of the upper limb. The overall trajectory of the exercise is in circular shape with predefined placement positions on its circumference. The trajectory is vertically divided into two parts: left and right for "Left Arm Training" and "Right Arm Training" respectively. When the user selects "Left Arm Training", only the objects from the left hand side will appear on its

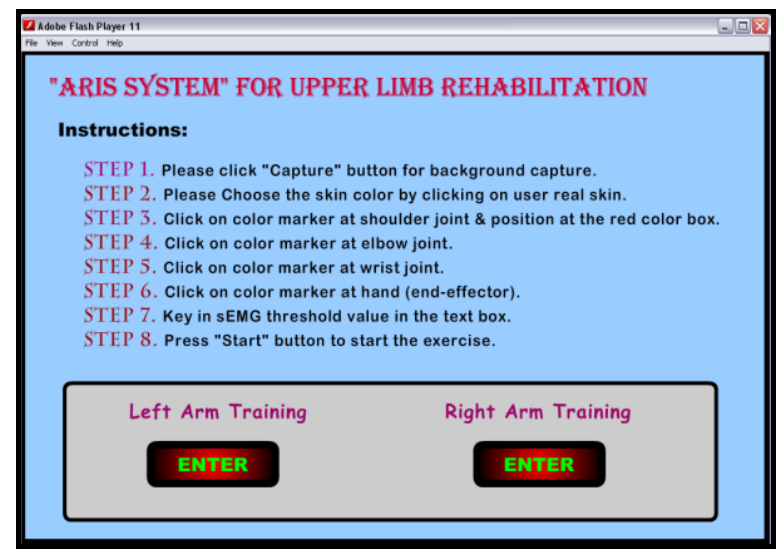

(a)

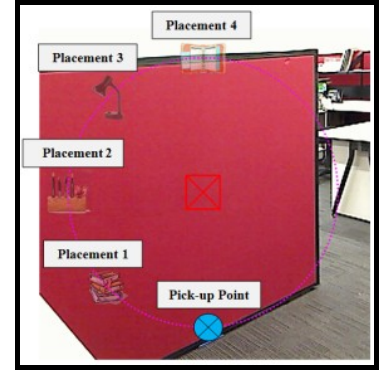

(b)

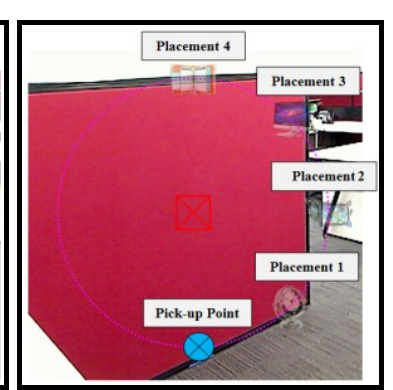

(c)
Figure 4. AR based rehabilitation exercise in ARIS (a) ARIS main page (b) Left Arm Training (c) Right Arm Training

circumference. When user selects "Right Arm Training", the objects from the right semicircle will display.

To perform the AIRS exercise, user requires to pick up the virtual object from pick up point and place that object at appropriate position on the display screen as shown in Figure 4(a) and 4(b). The placement positions are indicated as translucent objects for easy understanding. User needs to pick up one virtual object from pick up point at a time and there are total of four objects to pick up. The picking up of the virtual object is completed by the color marker at the finger tip which has already defined during the starting of the exercise. The picking is defined as a detection of collision between two objects: the color marker and the virtual object to be picked. Similarly, the placing of the virtual object is defined by the collision detection between translucent object and picked virtual object.

The ARIS exercise aims to induce the wider range of upper limb reaching movements such as shoulder flexion, abduction, adduction, internal and external rotation. The radius of the trajectory is fixed and the adjustment of the arm position on the display screen is adjusted manually.

\subsubsection{Real-time sEMG module}

The real-time sEMG signal is recorded by FlexComp acquisition device which is developed by Thought Technology Ltd. The signal is recorded with preamplified sEMG sensor which permitting the bandwidth 
of $20-500 \mathrm{~Hz}$ and the input range of $0-2000 \mu \mathrm{V}$. The raw sEMG data are first recorded via Matlab platform and then extracted with time domain feature extraction method called Root Mean Square (RMS) due to its easiness and good real-time information. The formula of the RMS is shown in equation (1).

$$
E M G_{r m s}=\sqrt{\frac{1}{N} \sum_{i=1}^{N} s E M G(i)^{2}}
$$

where sEMG(i) is the amplitude of the signal in $i^{\text {th }}$ sampling, $\mathrm{N}$ is the number of samples with the sampling rate of 2048. The extracted data are then imported into Flash professional platform in real time as an indication of user muscle activity. Based on this muscle activation level, the ARIS will detect the user intention to trigger the VA model simulation. Therefore, the activation of the VA model is subject-dependent according to the individual muscle activation level. It defines by therapist or carer during the training stage and only one signal which is recorded from anterior deltoid muscle as this signal is the most reflected signal for ARIS rehabilitation exercise as shown in Figure 5. However, all other three muscles activities are also recorded to observe and evaluate the muscle performance. After user has trained for some period, therapist will be able to observe the improvement of user upper limb activity according to the value of activation level.

\section{Materials and Method}

\subsection{Participants}

Seven participants with normal eyesight and sense of touch participated in the experiment. All the participants are right handed and all of them are students from University of Technology Sydney. Three participants were requested to perform "Left Arm Training" while the rest were performed "Right Arm Training".

\subsection{Setting and apparatus}

The experiment was conducted in one of the Lab rooms at Faculty of Engineering and Information Technology,

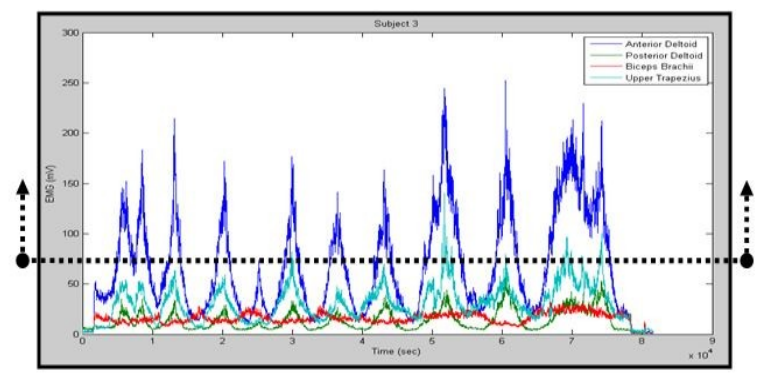

Figure 5. Example of sEMG activation level of subject 2 (in this case is 75)
University of Technology Sydney. To perform the experiment, participant was asked to sit in front of the desk where the personal computer with webcam and FlexComp sEMG acquisition device were placed. After that, four color markers were attached to the participant's arm as illustrated in Figure 2. The four sEMG electrodes were also attached to the respective positions which indicate with four green dots as shown in Figure 1. Subsequently, user was requested to move his/her arm in circular shape freely and activation levels of all muscles were recorded. After recording of activation level, the distance between the webcam and participant's arm position was adjusted via webcam. This is to align the size of VA model and real arm for the ownership illusion and define the distance for the real arm range of motion with respect to rehabilitation exercise. The adjustment was done by placing the real arm shoulder joint to the rectangular box (calibrating point) which is displayed at the centre of the rehabilitation exercise. After all the settings had done, participant was ready to perform the experiment.

\subsection{Experimental design}

To complete the experiment, it was separated into two phases: training phase and testing phase.

\subsubsection{Training phase}

During training phase, for every participant, the procedure was explained to familiar with the system and rehabilitation exercise. Afterwards, every participant was trained for one or two sessions on how to perform the upper limb rehabilitation exercise, what would be the measurements and what types of measurements should take note. The sEMG value was also observed during this stage to determine the appropriate muscle activation level as portrayed in Figure 5. This value was typed into the "Threshold Level" box to set as activation level where above this value, it was considered that participant had an intention to start the movement and therefore, VA model simulation was begun. For those participants who were novice in AR environment and illusion concept were provided more training from three to five sessions according to user self-confidence level. There was resting time between every training session to prevent from muscle fatigue. Only when the participant felt confident to perform independently, testing phase was conducted.

\subsubsection{Testing phase}

During testing phase, every participant was expected to perform the rehabilitation exercise independently which is exactly same as training sessions. The same value for the activation level from the last training session was set to determine the participant's intention of movement. In this phase, participant was asked to concentrate on the movement intention, feeling of ownership illusion as well as observing the real arm movement performance via 


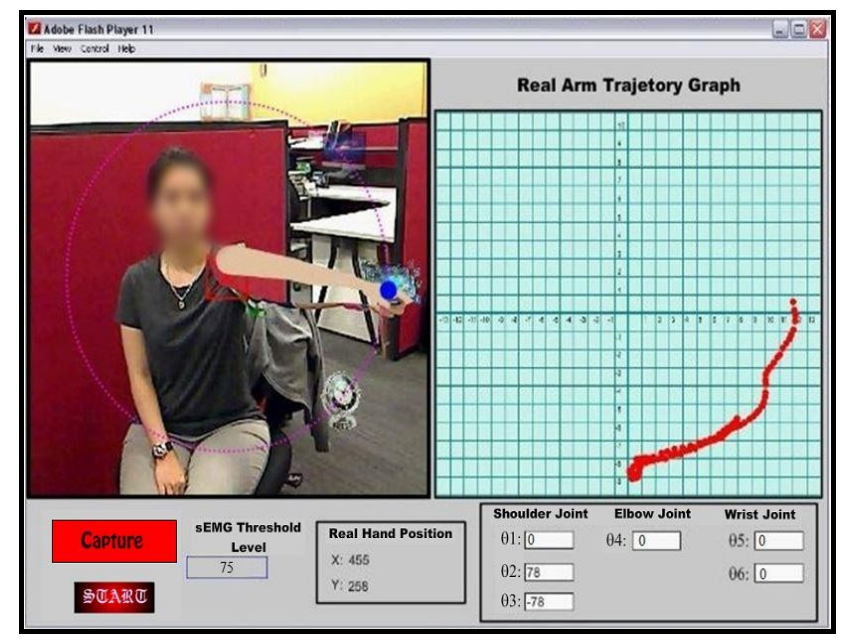

Figure 6. One of the participants performing during testing phase

trajectory graph and real-time current shoulder joint angle. The screen capture of testing phase is illustrated in Figure 6.

\subsection{Measurements}

In the current measurement, questionnaires which include the artificial visual feedbacks were assessed by every participant at the end of the testing phase. The answers for the questionnaires are in accordance with the visualanalogue likert scale [19] where ' 5 ' refers to strongly agree and ' 1 ' refers to strongly disagree. The set of questions that stated in the questionnaires are as follow:

1. I have Augmented Reality / Video Games experience.

2. The Graphical User Interface (GUI) is userfriendly and easy to adapt.

3. AIRS provide with enough information such as current hand position and joint angles.

4. During the training phase, I received enough information and training sessions.

5. The multiple colors tracking in ARIS is good.

6. AR environment was good and immersive.

7. AR exercise was doable and easy to perform. (Reaching movements)

8. The virtual objects in AR exercise were easy to pick up. (Collision detection)

9. During training and testing phase, I felt that when my real arm moved, virtual arm would move.

10. During training and testing phase, if the virtual arm moved, my real arm was moving.

11. During training and testing phase, I felt that virtual arm was my own arm.

12. During training and testing phase, I felt that I am picking up the virtual object by my own arm.

13. During training and testing phase, I felt that my upper limb muscles were fatigue.
14. The immediate feedbacks such as real-time trajectory tracking, threshold level, hand position and joint angles are very good and useful.

15. As a whole, the ARIS is interesting, motivating and enjoyable.

16. Suggestion and improvements are welcome!

Questions: 1 to 4 and 15 refer to the general questions that how did the participant feel about information, understanding and human-computer interface in ARIS. Questions: 5 to 8 refer to the development of AR environment and rehabilitation exercise. Questions: 9 to 12 refer to the ownership of the virtual arm. Question: 13, 14 and 16 refer to the feedback or response by the participants.

Other types of measurement that available in ARIS are real-time trajectory and threshold level. Up to current development stage, these two measurements are recorded manually for both training and testing phase by carer or therapist.

\section{Result Discussion}

The evaluation results attained by questionnaire are as portrayed in Figure 7, it can be clearly seen that all the participants were enjoyed and interested during the experiment. All the participants received adequate training sessions, information and procedure on how to perform and manipulate the rehabilitation exercise. Most of the participants were also found that multiple colors tracking technique that developed in ARIS was good. Most of the time, all the color markers were able tracked except sometimes in training phase, some color markers were out of webcam vision as some of the participants were not familiar with webcam applications and AR technology. They also felt that collision detection was good because it was very stable in picking and placing the virtual object. The exercise that developed in ARIS was also reported very ease of understanding and manipulation and also participants were felt the good sense of immersion in AR environment. The threshold level of

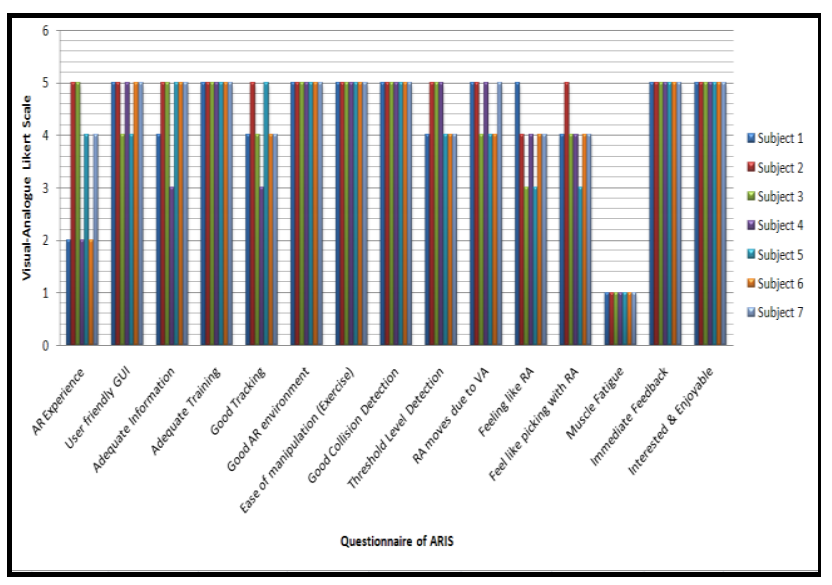

Figure 7. Questionnarie analysis result of the ARIS 
anterior deltoid that defined before training and testing phase was good and VA model was received real-time activation command to simulate the VA model. The feeling of ownership illusion was also evaluated via questionnaire and the responses from the participants were encouraging. Most of the participants were perceived as if real arm was moving. They also found that when they had intention to move their real arm, the virtual arm was moved at the same time. As a result, VA movement encouraged the real arm to move again like VA. As far as muscle fatigue was concerned, none of the participants were feel any muscle fatigue during training and testing phase as they were given enough rest between each session. Although most of the participants did not have AR experience, the training phase helped them to adapt the AR environment and illusion concept easily. This reflects on the real-time trajectory graph measurements. As far as trajectory graph measurement is concerned, the performances of two participants: subject 2 and subject 4 are illustrated in Figure 8. During the first training session, the movement trajectory that performed by subject 2 is scattered a lot as shown in Figure 8(a). However, the scatter range improved during the second training as the subject learned how to perform the exercise and able to control on his own movement as portrayed in Figure 8(b). This result shows that, at this stage, subject 2 achieved self-confidence to perform the next phase: testing phase. The trajectory result from testing phase was almost smooth semicircle which is an ideal trajectory curve for ARIS exercise as depicted in Figure 8(c). Similarly, performance of subject 4 is depicted in Figure 8 (d), (e) \& (f). After performing two training sessions, subject 4 was able to perform the exercise confidently although he was novice in AR environment. These results provide that the developed ARIS exercise is easy to understand and able to adapt quickly which will offer great benefit to the stroke patients. As far as threshold/activation level measurement is concerned, the value is almost consistent for individual in both training and testing phases as all the participants that involved in this preliminary stage had healthy muscle activities. This value will become one of the important measurements in ARIS when the system performs for clinical trials which will be conducted in near future.

Although, the overall questionnaire and graphical measurement results provided with positive feedback for ARIS, some of the participants were suggested very good ideas to improve in some parts of the system. For instance, instead of manual adjustment of the distance between webcam and participant sitting position to determine the radius of exercise trajectory, the length of participant's arm can be detected by webcam automatically. Another suggestion was to improve the appearance of the VA model by adding of curvatures and light effect to perceive as real arm like model.

As a preliminary stage of the development, it can be considered as a very successful system. The visual illusion technique was successfully introduced in ARIS and performance of real arm movement provided with promising results and feedbacks were able to access by everyone: users, carers or therapists. However, the appearance of the VA model will be improved by utilizing of specialized AR rendering API and imported into ARIS which is currently undertaking. As far as future work is concerned, blending with our previous developments [8] to provide the various choices of upper limb rehabilitation exercises and display of real-time activated muscle display. After upgrading has completed, new version of ARIS will be conducted more non-clinical trials and clinical trials at Port Kembla Hospital, New South Wales,

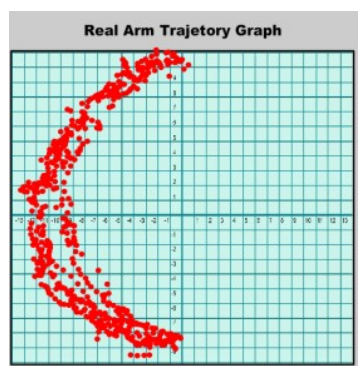

(a)

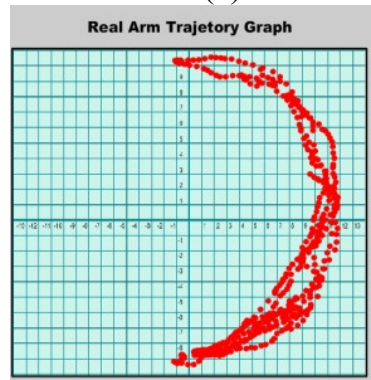

(d)

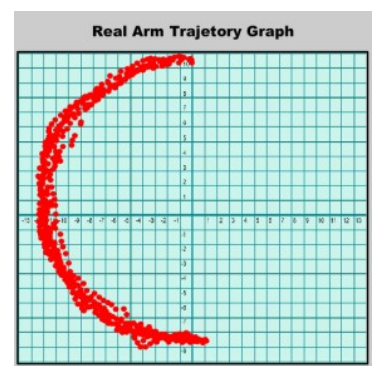

(b)

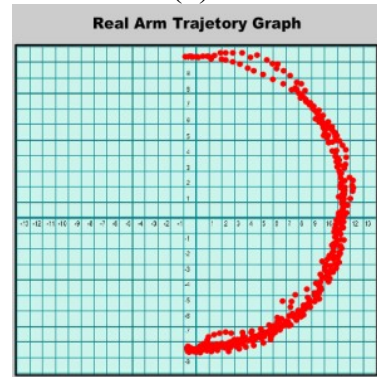

(e)

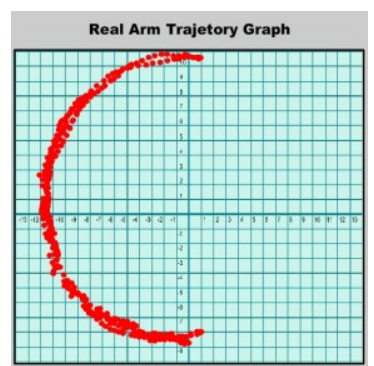

(c)

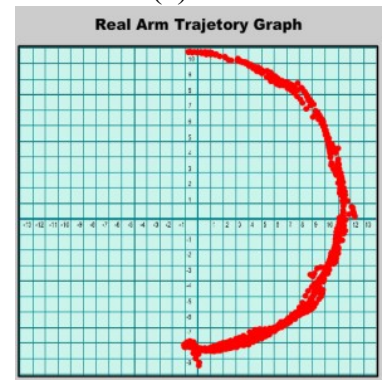

(f)

Figure 8. Results of own movement performance by subject 2 (a) during $1^{\text {st }}$ training, (b) during $2^{\text {nd }}$ training, (c) during testing phase with Left Arm Training and by subject 4 (d) during $1^{\text {st }}$ training, (e) during $2^{\text {nd }}$ training, (f) during testing phase with Right Arm Training 
Australia where the previous work of our group had demonstrated [20].

\section{Conclusion}

In this paper, real time biosignal-driven illusion system for upper limb motor rehabilitation named ARIS is proposed. The developed system is a low cost high motivation system and it can be used as both hospital and home-based therapy system with minimum supervision of therapists and trained carers. The system detects the user intention of movement via sEMG and simulates the virtual arm where the ownership illusion environment is induced. Left and right reaching exercise was chosen as an upper limb rehabilitation exercise for its wide range of motions. All the real-time feedbacks and measurements are able to access immediately by users, carers and therapists in proposed system. Experimental results have shown the effectiveness of the proposed system. As a preliminary stage of the development, it can be considered as a very successful system and has a potential as homebased upper limb rehabilitation system.

\section{References}

[1] National Stroke Foundation-Australia. (2014, March). Facts and Figures about Stroke. Available: https://strokefoundation.com.au/health-

professionals/tools-and-resources/facts-and-figures-aboutstroke/

[2] K. Kiguchi and Y. Hayashi, "An EMG-Based Control for an Upper-Limb Power-Assist Exoskeleton Robot," Systems, Man, and Cybernetics, Part B: Cybernetics, IEEE Transactions on, vol. 42, pp. 1064-1071, 2012.

[3] H.-D. Lee, B.-K. Lee, W.-S. Kim, J.-S. Han, K.-S. Shin, and C.-S. Han, "Human-robot cooperation control based on a dynamic model of an upper limb exoskeleton for human power amplification," Mechatronics, vol. 24, pp. 168-176, 2014.

[4] J.-H. Shin, H. Ryu, and S. H. Jang, "A task-specific interactive game-based virtual reality rehabilitation system for patients with stroke: a usability test and two clinical experiments," Journal of NeuroEngineering and Rehabilitation, vol. 11, p. 32, 2014.

[5] Y. M. Aung and A. Al-Jumaily, "Development of Augmented Reality Rehabilitation Games Integrated with Biofeedback for Upper Limb," in 5th International Convention on Rehabilitation Engineering \& Assistive Technology, Bangkok, Thailand, 2011.

[6] Y. M. Aung and A. Al-Jumaily, "Augmented Reality Based Reaching Exercise for Shoulder Rehabilitation," in 5th International Convention on Rehabilitation Engineering \& Assistive Technology, Bangkok, Thailand, 2011.

[7] M. Sha, M. Varley, S. Lik-Kwan, and J. Richards, "EMG Biofeedback Based VR System for Hand Rotation and Grasping Rehabilitation," in Information
Visualisation (IV), 2010 14th International Conference, 2010, pp. 479-484.

[8] Y. M. Aung and A. Al-Jumaily, "Augmented realitybased RehaBio system for shoulder rehabilitation," International Journal of Mechatronics and Automation, vol. 4, pp. 52-62, 2014.

[9] L. M. Vaca Benitez, M. Tabie, N. Will, S. Schmidt, M. Jordan, and E. A. Kirchner, "Exoskeleton Technology in Rehabilitation: Towards an EMG-Based Orthosis System for Upper Limb Neuromotor Rehabilitation," Journal of Robotics, vol. 2013, p. 13, 2013.

[10] B. C. Tsai, W. W. Wang, L. C. Hsu, L. C. Fu, and J. S. Lai, "An articulated rehabilitation robot for upper limb physiotherapy and training," in Intelligent Robots and Systems (IROS), 2010 IEEE/RSJ International Conference on, 2010, pp. 1470-1475.

[11] J. Foell, R. Bekrater Bodmann, M. Diers, and H. Flor, "Mirror therapy for phantom limb pain: Brain changes and the role of body representation," European Journal of Pain, 2014.

[12] J. Trojan, M. Diers, X. Fuchs, F. Bach, R. BekraterBodmann, J. Foell, S. Kamping, M. Rance, H. Maaß, and H. Flor, "An augmented reality home-training system based on the mirror training and imagery approach," Behavior Research Methods, pp. 1-7, 2013/12/13 2013.

[13] A. Reinersmann, J. Landwehrt, E. K. Krumova, J. Peterburs, S. Ocklenburg, O. Güntürkün, and C. Maier, "The rubber hand illusion in complex regional pain syndrome: Preserved ability to integrate a rubber hand indicates intact multisensory integration," PAIN, vol. 154, pp. 1519-1527, 2013.

[14] H. Regenbrecht, S. Hoermann, G. McGregor, B. Dixon, E. Franz, C. Ott, L. Hale, T. Schubert, and J. Hoermann, "Visual manipulations for motor rehabilitation," Computers \& Graphics, vol. 36, pp. 819. 834, 2012.

[15] C.-Y. Wu, P.-C. Huang, Y.-T. Chen, K.-C. Lin, and H.-W. Yang, "Effects of Mirror Therapy on Motor and Sensory Recovery in Chronic Stroke: A Randomized Controlled Trial," Archives of Physical Medicine and Rehabilitation, vol. 94, pp. 1023-1030, 2013.

[16] Thought Techonology. (2014, March). FlexComp Infiniti. Available: http:/www.thoughttechnology.com/

[17] Y. M. Aung and A. Al-Jumaily, "Illusion Approach for Upper Limb Motor Rehabilitation," in International Congress on Neurotechnology, Electronics and Informatics (NEUROTECHNIX 2013), Vilamoura, Algarve, Portugal, 2013, pp. 99-105.

[18] L. W. Pedretti, Occupational Therapy: practice skills for physical dysfunction, 2nd ed.: Mosby, 1985.

[19] H. Desurvire, M. Caplan, and J. A. Toth, "Using heuristics to evaluate the playability of games," in $\mathrm{CHI}$ '04 Extended Abstracts on Human Factors in Computing Systems, Vienna, Austria, 2004, pp. 1509-1512.

[20] Y. M. Aung and A. Al-Jumaily, "Shoulder rehabilitation with biofeedback simulation," in 2012 International Conference on Mechatronics and Automation (ICMA), Chengdu, China, 2012, pp. 974-979. 